Résumés des conférences et travaux

\title{
Littérature philosophique à Byzance et sa posterité à l'époque moderne
}

\section{Michel Cacouros}

\section{(2) OpenEdition}

\section{Journals}

Édition électronique

URL : https://journals.openedition.org/ashp/3552

DOI : $10.4000 /$ ashp.3552

ISSN : 1969-6310

Éditeur

Publications de l'École Pratique des Hautes Études

\section{Édition imprimée}

Date de publication : 1 septembre 2020

Pagination : 79-87

ISSN : 0766-0677

\section{Référence électronique}

Michel Cacouros, «Littérature philosophique à Byzance et sa posterité à l'époque moderne », Annuaire de l'École pratique des hautes études (EPHE), Section des sciences historiques et philologiques [En ligne],

151 | 2020, mis en ligne le 09 juillet 2020, consulté le 06 juillet 2021. URL : http:// journals.openedition.org/ashp/3552 ; DOI : https://doi.org/10.4000/ashp.3552 


\title{
LITTÉRATURE PHILOSOPHIQUE À BYZANCE ET SA POSTERITÉ À L'ÉPOQUE MODERNE
}

\author{
Maître de conférences : M. Michel Cacouros
}

Programme de l'année 2018-2019: Philosophie, disciplines littéraires et sciences à Byzance et dans l'après-Byzance: a. Les opuscules et corpus de logique dus à Jean Chortasménos (ca 1370-1431), étude et édition (suite); b. Tradition, exégèse et enseignement des Météorologiques d'Aristote à Byzance et dans l'après-Byzance, étude, avec édition de textes exégétiques.

\section{A. Les opuscules et corpus de logique dus à Jean Chortasménos (ca 1370-1431), étude et édition}

Ainsi qu'il avait été prévu, cette année nous avons continué l'étude du Corpus logicum chortasmenianum, en étudiant les trois concepts qui nous semblent prévaloir dans son origine et son organisation : 1. la reprise littérale, ou presque, de textes plus anciens (question liée à l'usage direct ou indirect des sources utilisées); 2. la création d'œuvres-citations; 3a-b. la production de nouveaux corpus scholiastiques basée sur le « mixage » du contenu entre les corpus disponibles (3a); sur celle-ci se greffe (3b) la création de nouveaux corpus scholiastiques de constitution codicologique mixte. Si le premier concept (1) est systématique auprès des auteurs byzantins, de même que (3a), celui du « mixage » du contenu des anciens corpus dans l'objectif d'en produire de nouveaux, en revanche, les deux autres, sur lesquels nous avons depuis plusieurs années attiré l'attention, semblent plus particuliers à Chortasménos; probablement sans en être l'auteur, celui-ci a dû les développer considérablement. Quant aux problèmes que pose l'édition du Corpus logicum chortasmenianum, ils sont directement liés à l'application de ces concepts et seront présentés l'année prochaine, de même par ailleurs que les autres questions relatives au Corpus.

1. Emprunts textuels dans les Opuscula chortasméniens. Le phénomène de la reprise de textes plus anciens est, on le sait, systématique à Byzance. Si cette manière de procéder est profondément ancrée dans la mentalité byzantine, c'est parce que les Byzantins ont fait du patrimoine grec antique, en particulier des textes dus aux Auctoritates, des modèles de style et de culture qu'il fallait à tout prix imiter ${ }^{1}$. Il en résulte que la citation devient le maître mot autour duquel s'organise la composition d'une œuvre; nous avons décrit cette situation dans un rapport précédent, au sein duquel nous attirions l'attention sur l'existence de cette " culture de la citation », qui s'applique également à la littérature exégétique d'Aristote ${ }^{2}$. Les emprunts textuels, ainsi

1. Parmi les recherches consacrées sur ce sujet, nous nous contentons de renvoyer à la contribution clas-

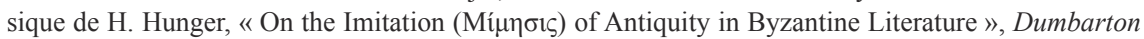
Oaks Papers, 23-24 (1969-1970), p. 17-38 = Id., Byzantinsche Grundlagenforschung. Gesammelte Aufsätze, Londres, 1973 (XV. Variorum Collected Studies 21).

2. M. Cacouros, « La culture de la citation et l'usage des florilèges », Annuaire. Résumés des conférences et travaux, $142^{e}$ année, 2009-2010, EPHE, PSL, SHP, Paris, 2011, p. 65-70. 
que nous l'avions souligné dans ce contexte, se présentent tantôt sous forme de citations (qui, dans plusieurs cas, ne sont pas signalées comme telles), tantôt sous forme d'extraits reproduits les uns à côté des autres. Le second cas de figure correspond aux corpus, le terme « corpus » étant utilisé dans le sens le plus général, qui couvre d'une part les florilèges et les travaux similaires, d'autre part les corpus scholiastiques; ces derniers sont composés d'extraits de différents commentaires (dont les auteurs sont en principe mentionnés en tête de chaque extrait) et / ou de scholies libres ${ }^{3}$. S'agissant de Chortasménos, les deux cas de figure précédemment évoqués sont amplement représentés : d'une part, il a produit des corpus composés d'extraits exégétiques, d'autre part ses Opuscula comportent de nombreux emprunts textuels, souvent non signalés comme tels ${ }^{4}$.

Un phénomène parallèle : usage direct ou indirect des sources utilisées? Avant de nous pencher sur la raison d'être des emprunts textuels au sein des Opuscula, il faut attirer l'attention sur le fait qu'ils posent, comme il se passe fréquemment dans la littérature exégétique à Byzance, un problème d'ordre différent, celui de l'usage direct ou indirect des sources reproduites sous forme d'extraits. Si la question se pose pour Chortasménos, c'est parce que, dans certains cas, les passages qu'il emprunte sont reproduits à l'identique par rapport aux sources premières, alors que, dans d'autres, ils ont manifestement été revus et modifiés soit par lui soit avant lui. $\mathrm{Si}$, dans le premier cas, il ne serait pas illégitime de penser qu'ils ont été directement empruntés à la source exégétique première, le doute s'impose dans le second cas. Tel est le cas, par exemple, de certains passages exégétiques reproduits dans les Prolégomènes I de Chortasménos. Souvent, il s'avère que Chortasménos a eu recours à des blocs exégétiques, comprenant des corpus d'extraits et / ou de scholies dans lesquels les extraits choisis avaient déjà été insérés, probablement depuis longue date, et modifiés au fil du temps. Dans d'autres cas, il pourrait être lui-même l'auteur des modifications textuelles. Bien entendu, la présence de ces extraits modifiés est hautement utile à l'établissement du texte des Opuscula, car, à moins de supposer que Chortasménos ait lui-même modifié les extraits choisis, elle oblige l'éditeur à localiser le chaînon intermédiaire manquant... en revanche, lorsqu'on n'observe pas des modifications de ce type, on reste dans le doute : en effet, absolument rien n'interdit qu'un passage exégétique soit reproduit à l'identique par rapport à la source première, sans pour autant

3. Ibid., p. 69-70; voir aussi Id., «L'enseignement des disciplines littéraires (Trivium), mathématiques (Quadrivium) et de la philosophie à Byzance : conception et organisation, pratiques de l'oral et techniques de l'écrit», dans les actes du colloque historique international De l'Antiquité à nos jours : histoire et méthodes de l'enseignement, organisé par M.-O. Munier, abbaye-école de Sorèze, 26-27 octobre 2006, publié aux Presses du centre universitaire Champollion, Albi, 2007, p. 1-83, et, en particulier, p. 73-79.

4. Ainsi, ils pourraient être considérés comme des «plagiats » : bien entendu, l'application de ce terme est anachronique pour Byzance; de plus, indépendamment du décalage temporel, ce terme traduit un état de pensée individuel qui n'a rien de commun avec les traits de la mentalité byzantine que nous venons de décrire. Quant aux deux cas de figure évoqués pour Chortasménos, leur étude dans le présent résumé et notre résumé précédent (« Les corpus et opuscules de logique dus à Jean Chortasménos (ca 1370-1431), étude et édition », Annuaire. Résumés des conférences et travaux, 150ªnnée, 20172018, EPHE, PSL, SHP, Paris 2019, p. 88-96) s'inspire de nos contributions « Jean Chortasménos et la culture du recueil » et « Chortasménos et Scholarios, le maîte et le disciple, face à la mentalité byzantine du recueil et du corpus », sous presse. 
provenir d'elle, mais d'un corpus scholiastique dans lequel il a été véhiculé sans avoir été altéré. La conclusion est que, d'une manière ou d'une autre, l'éditeur a souvent du mal à identifier le degré et le type d'éloignement qui séparent l'extrait cité par Chortasménos de l'original employé.

2. Le concept de l'ouvre-citation. Pour comprendre le traitement que Chortasménos réserve aux citations, nous avons eu recours au concept de l'œuvre-citation, qui devait lui être particulièrement familier, sûrement aussi à d'autres Byzantins. Par ce terme, nous entendons une œuvre conçue et réalisée par son auteur de manière à être constituée exclusivement ou majoritairement de citations; toutefois, il ne s'agissait pas de juxtaposer un ensemble d'extraits choisis, comme dans le cas d'un florilège, il fallait disposer les citations de manière à assurer l'unité de sens de l'ensemble ainsi obtenu; en d'autres termes, l'objectif était de produire une œuvre littéraire, non un florilège. Lorsque Chortasménos procède de la sorte, il fait preuve d'une maîtrise indéniable dans le choix des passages, leur agencement par juxtaposition et l'organisation des transitions. Il est même difficile de réaliser que le texte ainsi obtenu, qui, de plus, peut s'étendre sur plusieurs dizaines de pages, est issu d'un collage. Quant au contexte dans lequel s'insèrent les extraits empruntés par Chortasménos, il est souvent similaire au cadre d'origine, mais il peut aussi varier de manière conséquente.

Deux exemples suffiront. La Métaphrase de Chortasménos aux Seconds Analytiques, livre II, est en réalité une ouvre-citation, autrement dit elle est composée d'un ensemble de citations qui, majoritairement empruntées au commentaire de Philopon et de Prodrome à ce livre d'Aristote, sont juxtaposée avec dextérité les unes aux autres ${ }^{5}$. Dans ce cas, le contexte reste le même, à savoir l'exégèse du livre II des Seconds Analytiques. La situation est tout à fait différente lorsque Chortasménos reproduit comme nous l'avons signalé dans notre précédent rapport ${ }^{6}$, dans ses Prolé- $^{-}$ gomènes à la logique d'Aristote ${ }^{7}$, un extrait particulièrement étendu de l'Opusculum theologicum 12 de Néophytos Prodromènos, sans citer le nom de l'auteur concerné 8 . L'Opusculum 12 de ce moine philosophe et théologien, dont l'activité se situe dans

5. Étude de sa composition dans notre contribution « Un commentaire byzantin (inédit) au deuxième livre des Seconds Analytiques d'Aristote, attribuable à Jean Chortasménos», Revue d'histoire des textes, 24 (1994), p. 149-198, et, en particulier, p. 179-197. Voir les éléments supplémentaires fournis dans notre contribution « Jean Chortasménos, Théodore Prodrome et le Recueil de "Définitions" consacrées aux Seconds Analytiques, livre II. Contribution à l'étude de la tradition exégétique des Analytiques à

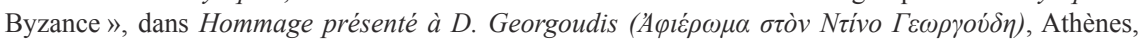
2007, p. 43-70, et, en particulier, p. 63-70 [rapports avec le commentaire de Théodore Prodrome et les Horoi aux Seconds Analytiques, livre II]. Cf. notre résumé précédent, « Les corpus et opuscules de logique dus à Jean Chortasménos (ca 1370-1431), étude et édition », p. 90, 95-96.

6. Nous les avons désignés comme Prolégomènes II, voir, parmi nos publications, ibid., p. 95.

7. Ibid.

8. Voir les références fournies, parmi nos publications, ibid., p. 95, n. 30-31. Numérotation de l'opuscule en question d'après mon édition de ses œuvres théologiques. Ainsi qu'il est signalé ibid., p. 95, Chortasménos emprunte aussi, dans le même contexte, des définitions provenant du lexique horistique de Néophytos, voir Id., « Le lexique des Définitions relevant de la philosophie, du Trivium et du Quadrivium compilé par Néophytos Prodromènos, son activité lexicographique et les corpus de textes philosophiques et scientifiques organisés par lui au monastère de Pétra à Constantinople ", dans L'erudizione scolastico-grammaticale a Bisanzio. Atti della VII Giornata di Studi Bizantini, Salerno, avril 2001, éd. P. Volpe-Cacciatore, Naples, M. D'Auria editore, 2003 (Dipartimento di Scienze dell'Antichità 
les dernières décennies du XIV ${ }^{\mathrm{e}}$ siècle, se réfère à la façon dont la lumière immatérielle et incréée qui entoura Jésus sur le Mont Thabor a pu être vue par ses disciples présents. Pour expliquer ce fait, l'auteur se réfère à la capacité de l'âme humaine de se représenter mentalement (phantasia) même les réalités qui sont insaisissables par la vue et, plus généralement, par les sens. En revanche, lorsque Chortasménos reprend le passage de Prodromènos dans les Prolégomènes à la logique d'Aristote, il l'insère dans un développement portant sur la capacité de l'âme humaine à syllogiser (à formuler des syllogismes). En effet, les Prolégomènes à la logique constituent en réalité une introduction aux Analytiques, Premiers et Seconds; c'est dans cette perspective que Chortasménos essaie de cerner le mécanisme syllogisant de l'âme humaine et de situer au sommet des facultés de l'homme la capacité de syllogiser, qu'il juge supérieure au simple raisonnement et, à plus forte raison, à la phantasia et à la sensation.

3a-b. Corpus scholiastiques par " mixage »; corpus scholiastiques de constitution codicologique mixte : le cas du Lovaniensis. À Byzance, lorsqu'on voulait produire un nouveau corpus, en l'occurrence exégétique, on avait l'habitude de "mixer ", à des degrés variables suivant les priorités établies, le contenu des corpus disponibles; le plus souvent, on choisissait un seul corpus comme principale ressource et c'est dans celui-ci qu'on introduisait les unités venant des autres corpus qu'on avait consultés. Cette pratique, qui était courante à Byzance et qui concerne uniquement le contenu, a également été appliquée par Chortasménos. Ainsi, dans notre précédent résumé, nous avions distingué, du point de vue du contenu, trois types de corpus qu'il a produits et utilisés, commentés et annotés : «a. les corpus qu'il a compilés lui-même; b. les corpus plus anciens qu'il a repris à son compte et réécrits en les remaniant de manière conséquente; c. les corpus plus anciens qu'il a annotés dans les manuscrits anciens avec des scholies marginales, dont il était souvent l'auteur, doublant ainsi les corpus anciens d'un corpus plus moderne $»^{9}$.

Tel est le cas du corpus exégétique qu'il a lui-même organisé dans le Lovaniensis ${ }^{10}$; il s'agit, rappelons-le, d'un manuscrit exégétique constitué d'une cinquantaine de folios non reliés, majoritairement écrits par Chortasménos ${ }^{11}$, que nous avons été appelé à examiner dès son achat en 1992 par le De Wulf-Mansion Centrum de la Katholieke Univeristeit de Leuven; depuis, nous avons eu l'occasion de l'examiner à plusieurs reprises et de lui consacrer un certain nombre d'études, faites dans le cadre de la monographie détaillée que nous lui consacrons ${ }^{12}$. Or, dans la partie du Lovaniensis écrite par Chortasménos ont été insérés, comme nous l'avons signalé à plusieurs reprises, « un demi-folio (partie sup.) et un bifeuillet qui sont plus anciens $\left(\mathrm{XIII}^{\mathrm{e}}-\mathrm{XIV}^{\mathrm{e}} \text { s.). }\right\rangle^{13}$; ceux-ci proviennent des deux manuscrits différents que nous avons pu localiser, le second étant le Princeton 173, de la fin du XIII ${ }^{\mathrm{e}}-$ début du

dell'Università degli Studi di Salerno, Associazione Italiana di Studi Bizantini), p. 165-220, et, en particulier, p. 181-184.

9. Citation de notre précédent résumé, «Les corpus et opuscules de logique dus à Jean Chortasménos (ca 1370-1431), étude et édition », p. 91.

10. Ibid., p. 91-93, avec mention de nos précédentes publications, p. 91 n. 13.

11. Voir ibid., p. 91 n. 13 sur l'identification de sa main.

12. Ibid., p. 91 et n. 13 .

13. En dernier lieu, ibid., p. 91-93. 
$\mathrm{XIV}^{\mathrm{e}}$ siècle ${ }^{14}$. Dans le sens inverse, dans l'actuel Princeton 173 a été inséré le f. 173A : celui-ci correspond, comme nous l'avons également démontré, à un folio qui, écrit par Chortasménos, provient du Lovaniensis; en effet, lors des examens successifs du Lovaniensis, nous avions conjecturé l'existence de ce folio alors perdu et nous en avions reconstitué le contenu, en le rattachant au dossier III du Lovaniensis (nous lui avions attribué le $\mathrm{n}^{\circ} \mathrm{f}$. IIIb) ${ }^{15}$. La conclusion est qu'un folio du Lovaniensis a été déplacé vers le Princeton 173 (peut-être on en avait déplacé davantage à l'origine) et que, dans le sens inverse, des folios de ce dernier et d'un autre manuscrit de la même époque ont été déplacés au Lovaniensis ${ }^{16}$.

Ces transferts reflètent, dans tous les cas évoqués, le même type de comportement de la part de leur auteur; ainsi, ils permettent, déjà, de trancher sur l'usage des folios déplacés : ceux-ci ont été délibérément introduits dans des manuscrits hôtes (autrement dit ceux qui recevaient les folios extérieurs) pour enrichir ces derniers et permettre une lecture exégétique faite en parallèle. Dans l'état actuel, le Lovaniensis constitue un " corpus scholiastique de constitution codicologique mixte» et il en est de même pour le Princeton 173 (y compris le folio actuellement désigné comme Princeton 173A). Par ce terme, nous entendons les corpus scholiastiques composés d'éléments codicologiques peu étendus (demi-folios, folios ou bifeuillets) provenant de manuscrits de date différente. Les corpus scholiastiques de constitution codicologique mixte ainsi produits sont factices, dans la mesure où ils sont dépourvus d'une vraie unité codicologique. De plus, certaines conditions étaient indispensables pour la production de ce type de corpus, permettant d'une part d'enlever certains folios à un manuscrit, d'autre part de les rattacher à un autre. Entre autres, il fallait que ces manuscrits ne fussent pas reliés ou, si ceci n'était pas le cas, il fallait que leur reliure fût défaite ou en mauvais état ${ }^{17}$. À ce titre, le Lovaniensis n'avait pas été relié, ce qui permettait d'y insérer des folios provenant d'autres manuscrits, mais, aussi, de retirer certains folios originaux, comme dans le cas du f. IIIb; quant à la reliure des manuscrits émetteurs que Chortasménos a employés, elle était probablement en mauvais état, ce qui lui a permis d'enlever un certain nombre de folios (sans doute ceux qu'il avait jugés comme les plus intéressants du point de vue de l'exégèse, ou les plus faciles à enlever) pour les insérer dans le Lovaniensis.

Est-ce bien Chortasménos qui est à l'origine de ces transferts de folios? Certes, il est difficile d'avoir une réponse sûre, étant donné d'une part le temps passé depuis l'époque où Chortasménos utilisait ces manuscrits, d'autre part leur état actuel. Il faut toutefois noter que tous les indices conservés font penser qu'il avait lui-même procédé à ces transferts, en tout cas à la plupart d'eux. En effet, nous avons déjà relevé le même type de comportement dans les transferts précédemment évoqués. De plus, les feuillets du Lovaniensis datant du XIII $-\mathrm{XIV}^{\mathrm{e}}$ s. et, également, le Princeton 173 portent des notes autographes de Chortasménos; elles montrent qu'il avait consulté tous ces manuscrits et qu'ils faisaient très probablement partie, comme nous l'avons

14. Ibid., p. 92 et n. 21.

15. Ibid., p. 92.

16. Ibid., p. 92-93.

17. Voir les cas de figure abordés ibid., p. 92. 
signalé, de la bibliothèque du monastère de Pétra à Constantinople ${ }^{18}$. Précisons que les feuillets qui, dans l'actuel Lovaniensis, proviennent de l'autre manuscrit du XIII ${ }^{\mathrm{e}}$ $\mathrm{XIV}^{\mathrm{e}}$ siècle que nous avions identifié présentent la même spécificité et montrent que ce témoin se trouvait probablement, lui aussi, dans la même bibliothèque. L'objectif de Chortasménos était d'ordre pratique : il voulait disposer à la fois de deux manuscrits qui étaient dans une forme non reliée ou fragmentaire et qui, à ce titre, étaient plus faciles à consulter. Ainsi que nous l'avions signalé, cette méthode lui permettait par exemple, de consulter " en parallèle, et de manière pour ainsi dire "fusionnelle", les actuels Lovaniensis et Princeton $173 »{ }^{19}$. Il faut aussi noter que Chortasménos se servait probablement, simultanément, de l'autre manuscrit du XIII ${ }^{\mathrm{e}}-\mathrm{XIV}^{\mathrm{e}} \mathrm{s}$. dont il a emprunté des folios, et que d'autres manuscrits encore faisaient sans doute partie du même ensemble.

Étant donné ces remarques, il est clair que l'ensemble ainsi constitué formait une bibliothèque légère, mobile et usuelle; il est également clair que les manuscrits en question avaient été choisis et aménagés non pas en fonction du critère codicologique, mais de leur contenu, dans l'objectif d'assurer un enseignement lors duquel le maître disposerait simultanément de tous les éléments requis, sans égard à leur origine codicologique. Cette bibliothèque mobile devait aussi lui permettre une consultation plurielle et simultanée de tous les éléments dont il avait besoin pour réviser de manière rapide et pratique ses notes, une sorte de bibliographie mobile, disponible à tout moment. Dans ces circonstances, avec un objectif si précis, force est de déduire que, très probablement, Chortasménos lui-même est à l'origine des transferts en question.

\section{B. Tradition, exégèse et enseignement des Météorologiques d'Aristote à Byzance et dans l'après-Byzance, étude, avec édition de textes exégétiques}

L'étude présentée dans cette partie du séminaire, qui devra s'échelonner sur trois à cinq ans, porte sur la tradition, l'exégèse et l'enseignement des Météorologiques d'Aristote dans l'Antiquité tardive (plus précisément néoplatonicienne) et à Byzance, de même que dans la période qui, depuis les travaux de N. Iorga, est désignée comme post-byzantine.

1. L'exégèse néoplatonicienne (tradition grecque). Cette année, nous avons commencé avec l'exégèse antique des Météorologiques, notamment avec les commentaires anciens de cet ouvrage d'Aristote conservés en tradition grecque, d'Alexandre d'Aphrodise (190-250 apr. J.-C.) ${ }^{20}$, de Philopon (ca 490-ca 570 apr. J.-C.) $)^{21}$ et

18. Ibid.

19. Ibid., p. 93.

20. Dates d'après R. Goulet, M. Aouad, Notice «Alexandros d'Aphrodisias », dans R. Goulet (éd.), P. Hadot (préface), Dictionnaire des philosophes antiques, t. I (d'Abam(m)on à Axiothéa), Paris, CNRS Éditions, 1989, p. 125-139. Dernière édition: Alexandri Aphrodisiensis In Aristotelis Meteorologicorum libros commentaria, éd. M. Hayduck, Berlin, 1899 (Commentaria in Aristotelem graeca, III, 2).

21. Dates d'après R. Sorabji, « John Philoponus », dans Id. (éd.), Philoponus and the rejection of Aristotelian science, Londres, Duckworth \& Co., 1987, p. 1-40, en particulier, p. 37. Dernière édition : Ioannis Philoponi In Meteorologicorum liber primum commentarium, éd. M. Hayduck, Berlin 1897 (Commentaria in Aristotelem Graeca XIV, 1). 
d'Olympiodore (495/505 - peu après 565) ${ }^{22}$. Sur cet ensemble, les deux commentaires néoplatoniciens ont particulièrement retenu notre attention. En nous fondant sur les travaux de nos prédécesseurs (A.-J. Festugière, L. G. Westernik, H.-D. Saffrey, A.-P. Segonds, I. Hadot, P. Hoffmann), nous avons présenté le contexte dans lequel ceux-ci ont vu le jour et la place occupé par les Météorologiques dans le cursus en cours au sein de l'école néoplatonicienne. En effet, les ouvrages aristotéliciens y étaient regroupés en sections thématiques, au sein desquelles ils occupaient une place précise; les Météorologiques faisaient partie de la section physique, où elles occupaient la quatrième position, après l'Audition physique, le Du ciel, le De la génération et de la corruption. Nous avons également passé en revue les éléments doctrinaux différenciant, d'après Aristote, les Météorologiques du traité $\mathrm{Du}$ ciel, le premier ouvrage aristotélicien portant sur l'espace sublunaire, l'autre sur l'espace céleste. En effet, ces éléments réglementent, pour ainsi dire, l'exégèse consacrée à chacun d'eux; quant à la partie occupée par les phénomènes ignés, malgré le fait qu'elle relève du monde sublunaire, elle constitue en réalité une zone de transition permettant le passage de ce monde vers l'espace supralunaire (cette zone, dans laquelle se meuvent les comètes, retiendra ultérieurement notre attention).

Les choix thématiques majeurs proposés par les exégètes néoplatoniciens et l'organisation de leurs exégèses ont également retenu notre attention. En effet, le commentaire d'Olympiodore est divisé en Praxeis, correspondant à des séquences thématiques liées à l'enseignement des Météorologiques, alors que le commentaire de Philopon, en tout cas la partie conservée de celui-ci, est différemment agencée. De plus, pour chaque entité thématique, Olympiodore applique en premier lieu une approche globale (théôria), puis un examen littéral, mot par mot (lexis), mais les limites entre les deux types d'approche sont encore imprécises chez Philopon, qui ne semble pas obéir au système de l'examen par théôria et lexis, ou se servir d'un système plus archaïque et moins performant. Nous nous sommes également penchés sur la tradition manuscrite de ces commentaires, tous les deux ayant été transmis de manière lacunaire (le commentaire de Philopon va uniquement jusqu'à Météor., I, 12, 348b34, alors que celui d'Olympiodore présente de nombreuses lacunes et erreurs ${ }^{23}$ ). En dernier lieu,

22. Dates d'après : The Greek Commentaries on Plato's Phaedo, vol. 1. Olympiodorus, éd. L. G. Westerink, Platonic Texts and Translations, vol. II, Amsterdam, North Holland Publishing, 1976; Wiltshire (UK), The Prometheus Trust, 2009 ( $2^{e}$ éd.), p. 20-21; K. Verrycken, « The development of Philoponus' thought », dans R. Sorabji (éd.), Aristotle Transformed: The ancient commentators and their influence, Londres, Duckworth \& Co, 1990, p. 242 n. 52; L. G. Westerink, « The Alexandrian commentators and the introductions to their commentaries ", ibid., p. 325-348, en part. p. 328-329=Anonymous Prolegomena to Platonic Philosophy, éd. L. G. Westerink, Platonic Texts and Translations, vol. V, Amsterdam, North Holand Publishing, 1962; réimprimé avec des corrections, Wiltshire (UK), The Prometheus Trust, 2011, p. XIII-XIV = Prolégomènes à la philosophie de Platon, texte établi par L. G. Westerink et traduit par J. Trouillard, avec la collab. de A.-P. Segonds, Paris, Les Belles Lettres, 1990 (CUF), p. XVII ; H.-D. Saffrey, « Olympiodoros d'Alexandrie », dans R. Goulet (éd.), Dictionnaire des philosophes antiques, t. IV (de Labeo à Ovidius), Paris, CNRS Éditions, 2005, p. 769-772, et, en part., p. 769. Dernière édition du commentaire : Olympiodori In Aristotelis Meteora commentaria, éd. G. Stüve, Berlin, 1900 (Commentaria in Aristotelem graeca XII, 2).

23. Sur les 53-54 praxeis qui constituaient probablement le commentaire, 47 au total ont été conservées, avec perte des praxeis $\kappa \theta^{\prime}-\lambda \beta^{\prime}\left[\mathrm{n}^{\text {os }} 29-32\right]$ et des 2-3 qui suivaient la dernière praxis transmise, $v \alpha^{\prime}$ $\left[n^{\circ} 51\right]$. 
nous avons abordé les différences marquantes qui séparent les deux commentaires. En effet, tous les deux devraient en principe faire état des mêmes positions doctrinales, qui étaient celles de l'école néoplatonicienne; si cette mise en parallèle s'applique vis-à-vis d'Ammonios (que Philopon a eu comme maître à l'école et que les deux commentateurs citent à plusieurs reprises) et des sources, en particulier astrométéorologiques, qui sont les mêmes dans les deux textes, elle présente des divergences du point de vue doctrinal. Celles-ci sont certainement dues au caractère contestateur de Philopon et, surtout, au fait qu'il était chrétien et que, à ce titre, certaines positions aristotéliciennes et, par la suite, néoplatoniciennes ne pouvaient pas être retenues ${ }^{24}$. Les positions proposées par Alexandre d'Aphrodise sont souvent âprement contestées par Philopon, alors qu'Olympiodore les considère de manière plus neutre. L'interprétation de ce dernier est purement néoplatonicienne et repose souvent sur l'autorité de Damascius. Notre intérêt a aussi été retenu par la systématisation qui caractérise le commentaire d'Olympiodore et les différents aspects que celle-ci revêt.

2. La tradition exégétique byzantine. Après la partie néoplatonicienne nous avons abordé le volet byzantin, peu ou insuffisamment étudié jusqu'à présent. D'après nos recherches, les Météorologiques ont été reçues à Byzance de trois manières différentes, dont chacune correspond à une étape différente dans la vie de l'empire. Lors de la première étape (qui va du $\mathrm{VI}^{\mathrm{e}} / \mathrm{VII}^{\mathrm{e}}$ au $\mathrm{VIII}^{\mathrm{e}} / \mathrm{IX}^{\mathrm{e}}$ siècle), les Byzantins se sont contentés de reprendre les commentaires antiques consacrés aux Météorologiques, sans procéder eux-mêmes à l'élaboration de nouvelles exégèses. La seconde ( $\mathrm{VIII}^{\mathrm{e}} /$ $\mathrm{IX}^{\mathrm{e}}-\mathrm{XI}^{\mathrm{e}} / \mathrm{XII}^{\mathrm{e}} \mathrm{s}$.) correspond à la formation de ce que nous avons appelé la «vulgate byzantine »; durant celle-ci, les Byzantins ont choisi un certain nombre d'éléments provenant des Météorologiques, qu'ils ont combinés avec d'autres venant du traité aristotélicien $\mathrm{Du}$ ciel et d'autres ouvrages, produisant ainsi un ensemble de contenu astrométéorologique qui était d'orientation cosmologique. Toujours dans la même perspective, ils y ont parfois ajouté d'éléments d'ordre théologique, renvoyant à la création de l'univers par le Dieu unique chrétien. Quant à la dernière étape $\left(\mathrm{du} \mathrm{XII}{ }^{\mathrm{e}} /\right.$ $\mathrm{XIII}^{\mathrm{e}}$ siècle à la chute de Byzance en 1453), les Byzantins interprètent désormais les Météorologiques dans le cadre d'une exégèse portant sur l'ensemble du Corpus, autrement dit sur toutes les œuvres du Stagirite (en tout cas celles qui étaient considérées comme telles à l'époque), chacune d'elles étant disposée suivant l'ordre thématique qu'elle occupait dans l'organisation néoplatonicienne du Corpus.

Notre examen du volet byzantin a commencé par un aperçu sur l'exégèse de l'ensemble du Corpus aristotelicum à Byzance, faite sous l'angle des rapports qu'elle entretient avec l'exégèse néoplatonicienne. En effet, le fonds exégétique byzantin repose souvent sur les textes exégétiques néoplatoniciens, il en est par conséquent largement tributaire. Les Byzantins ont souvent copié ces textes dans les manuscrits sans éprouver le besoin, au moins pendant les premiers siècles

24. Ainsi, dans son commentaire aux Météorologiques, Philopon essaie de minimiser le rôle de l'éther, proposant une lecture chrétienne de la théorie aristotélicienne du cinquième élément; voir K. Verrycken, "The development of Philoponus" thought», p. 258; L. G. Westerink, "The Alexandrian commentators and the introductions to their commentaries », p. 327-328; Prolégomènes à la philosophie de Platon, éd. L. G. Westerink, trad. J. Trouillard, avec la collab. de A. P. Segonds, p. XIv ; cf. R. Sorabji, « John Philoponus », p. 24-26. 
de Byzance, de produire des commentaires byzantins; suivant nos travaux, Michel d'Éphèse (fin du XI ${ }^{\mathrm{e}}$ - début du XII ${ }^{\mathrm{e}} \mathrm{s}$.) est le premier qui décide de doter ses contemporains d'un corpus exégétique byzantin portant sur l'ensemble du Corpus aristotelicum. Il en résulte que les Byzantins se sont toujours inspirés dans leurs propres travaux des modèles exégétiques néoplatoniciens et qu'ils en reproduisent souvent des extraits entiers. Nous avons aussi rappelé la variété des formes qui caractérise l'exégèse byzantine; ce sujet a retenu notre attention à plusieurs reprises, y compris au sein de notre thèse d'habilitation (2014).

Après cet aperçu, nous nous sommes penché sur l'exégèse des Météorologiques à Byzance, en présentant brièvement la tripartition précédemment exposée et en mentionnant, pour chaque étape, les choix thématiques majeurs, les sources exégétiques principales et les formes exégétiques pratiquées. À l'issue de cette présentation d'ordre global, nous sommes passé à l'étude de chaque étape, en commençant par les premières traces conservées d'une exégèse des Météorologiques à Byzance et les prémisses de la formation de la vulgate byzantine. Nous avons passé en revue un corpus scholiastique dont les scholies doivent remonter à cette époque, de même que le développement horistique du chapitre $\xi \eta^{\prime}$ [68] de la recensio brevior de la Dialectica, attribuée à Jean Damascène ( $c a$ 655-ca 745) $)^{25}$, en en localisant les sources. Nous avons entamé l'étude de l'œuvre météorologique de Michel Psellos, qui offre plusieurs exemples de l'utilisation de la vulgate byzantine, malgré les épineux problèmes d'attribution que plusieurs d'entre eux posent.

25. Édition du texte dans Die Schriften des Johannes von Damaskos, éd. P. B. Kotter, vol. 1. Dialectica (Capita philosophica), Berlin, Walter De Gruyter, 1969 (Patristische Texte und Studien 7), p. 140, 1. 1-p. 142, 1. 68. 